\title{
Free flap harvest from the infraumbilical region for head and neck reconstruction purposes: a retrospective cohort study.
}

Giordana Bettini ${ }^{1}$, giorgia saia ${ }^{2}$, Piero Franco ${ }^{2}$, Stefano Valsecchi, Laura Tognin ${ }^{2}$, Enrico Alexandre, Alberto Bedogni ${ }^{2}$

1 University-Hospital of Padova

2 University of Padua

Funding: The author(s) received no specific funding for this work.

Potential competing interests: The author(s) declared that no potential competing interests exist.

\section{Abstract}

The abdominal wall has showed many advantages as a donor site in head and neck reconstruction given its constant pattern of dominant perforators. Currently, standard design of the skin component of composite soft-tissue flaps recommended for head and neck reconstruction encompasses the lower and upper periumbilical region thus increasing the overall vascularization of the flap. We report on the safety of the harvest of abdominal soft-tissue free flaps from the infraumbilical region.

A retrospective charts review was performed. Consecutive subjects who underwent microsurgical reconstruction of composite defects of the Head \& Neck with infraumbilical free flaps between January 2008-January 2020 were enrolled. After surgery, patients were followed up at three-month intervals to evaluate the incidence of complications and the long-term outcome at the donor site.

25 patients underwent reconstruction with free flaps harvested from the lower abdomen. A total of 35 abdominal flaps (20 VRAM, 15 DIEAP) were performed; ten patients underwent bilateral flap harvest and abdominoplasty was performed to achieve direct closure.

Marginal abdominal skin necrosis occurred in 3 patients postoperatively. Abdominal bulge occurred in one case; neither hernia nor abdominal wall weakness were not encountered at a median follow-up of 12 months (IQR 6; 27).

The results of the study confirm that the infraumbilical region is a safe donor area of free flaps for head and neck reconstruction that preserves abdominal wall firmness and improves ultimate functional and aesthetic outcomes at the donor site.

\section{Definitions}

Abdominal Region

Defined by National Cancer Institute

Head and Neck

Defined by National Cancer Institute

Periumbilical Region

Defined by National Cancer Institute 


\section{Retrospective Study \\ Defined by National Cancer Institute \\ Disease-Free Survival \\ Defined by National Cancer Institute \\ Visual Analog Scale \\ Defined by National Cancer Institute \\ Impairment \\ Defined by National Cancer Institute}

\section{INTRODUCTION}

The abdominal wall has become an increasingly popular donor site for head and neckreconstruction. Several studies demonstrated the feasibility of different perforator flaps from the abdominal region as a suitable donor site to well fit defects with varied sizes and shapes in head and neck reconstruction. ${ }^{[1][2][3]}$

Usually, the skin component of Transverse/Vertical Rectus Abdominis myocutaneous flap (TRAM/VRAM) and Deep Inferior Epigastric Artery Perforator (DIEAP) flap used for reconstructive purpose in the cervico-facial area, is selectively designed covering the periumbilical area of the lower and upper abdomen to include the dominant perforators, hence harvesting highly vascularized flaps. ${ }^{[4]}$

Initially, it was a common belief that multiple perforators provided better vascularization of the flap. Various studies instead proved that flap survival is mainly determined by the distance between the main perforators and the midline. The latter statement is particularly true when considering viability of zone 4. ${ }^{[5]}$

Therefore, the flap is now routinely pedicled by only one or two medial row perforators that emerge from the medial branch of the deep inferior epigastic artery in the paraumbilical area, where the skin component is safely designed. [4][6] Different skin paddle designs extending over the entire abdominal wall (vertically, horizontally or obliquely oriented) have been successfully described to increase the amount of viable tissue harvested based on periumbilical perforators. ${ }^{[7]}[8]$ However, in case of large defects requiring bilateral flaps, the harvest of a paraumbilical skin paddle is generally associated with poor aesthetic outcomes. These result from worse abdominal contour and a less concealable scar when compared to the harvest of a flap in the inferior aspect of the abdomen. ${ }^{[7]}$

We report on the safety of the harvest of abdominal soft-tissue free flaps from the infraumbilical region.

\section{PATIENTS AND METHODS}

\section{Study design and setting}

A multicenter retrospective cohort study on consecutive patients who underwent reconstruction of composite defects of the Head and Neck region with infraumbilical abdominal free flaps was performed at the Units of Maxillofacial Surgery of Verona and Padua (Italy). All subjects signed informed consent to be enrolled in the present study. The institutional ethical 
committee approved the study.

\section{Patients}

Consecutive subjects who underwent primary or delayed reconstruction of major head and neck soft tissue defects with abdominal perforator free flaps from the infraumbilical area were eligible for the study. Patients were excluded in presence of previous history of surgical violation of the anterior abdominal wall for other disorders.

\section{Data collection and variables}

The clinical charts of the patients treated at our Units between January 2008 and January 2020 were reviewed. Relevant clinical data were extracted and entered into an electronic spreadsheet form. The following data were collected from the clinical charts: age, sex, reason for surgery (diagnosis), date of surgery, type of flap harvest, side of the flap harvest, size of the skin flap component, type of abdominal wall closure; cervico-facial defect type and size were also recorded.

\section{Surgery}

All cases treated at the recruiting centers had been routinely discussed at the institutional multidisciplinary head and neck oncology board that included the oncologist, radiotherapist, the radiologist and the head and neck surgeons. Thereafter, the ablative and reconstructive treatment options were assessed by experienced head and neck microsurgeons (A.B., S.V.) and discussed with the patients and their relatives. Surgical procedures were performed by the same operators (A.B., S.V.) in all cases.

During preoperative examination, periumbilical perforators were detected using a Doppler probe and marked on the abdominal skin.

At the time of surgery, linear dimensions of the planned defect size and shape were preoperatively assessed to properly mark the area of the skin component of the flap on the lower aspect of the abdomen. High-definition intraoperative pictures of the planned abdominal flap design were obtained after skin marking.

The skin paddle was designed in the lower abdomen based on periumbilical perforators in the area beneath the umbilicus. For the unilateral harvest of flaps, the abdominal skin paddle was oriented vertically. Typically, the skin island is comprised between the umbilicus and the suprapubic crease. In muscle-containing flap (e.g. VRAM), the skin paddle was centered over the carrier rectus muscle (Fig. 1A-B; Fig. 2A) 


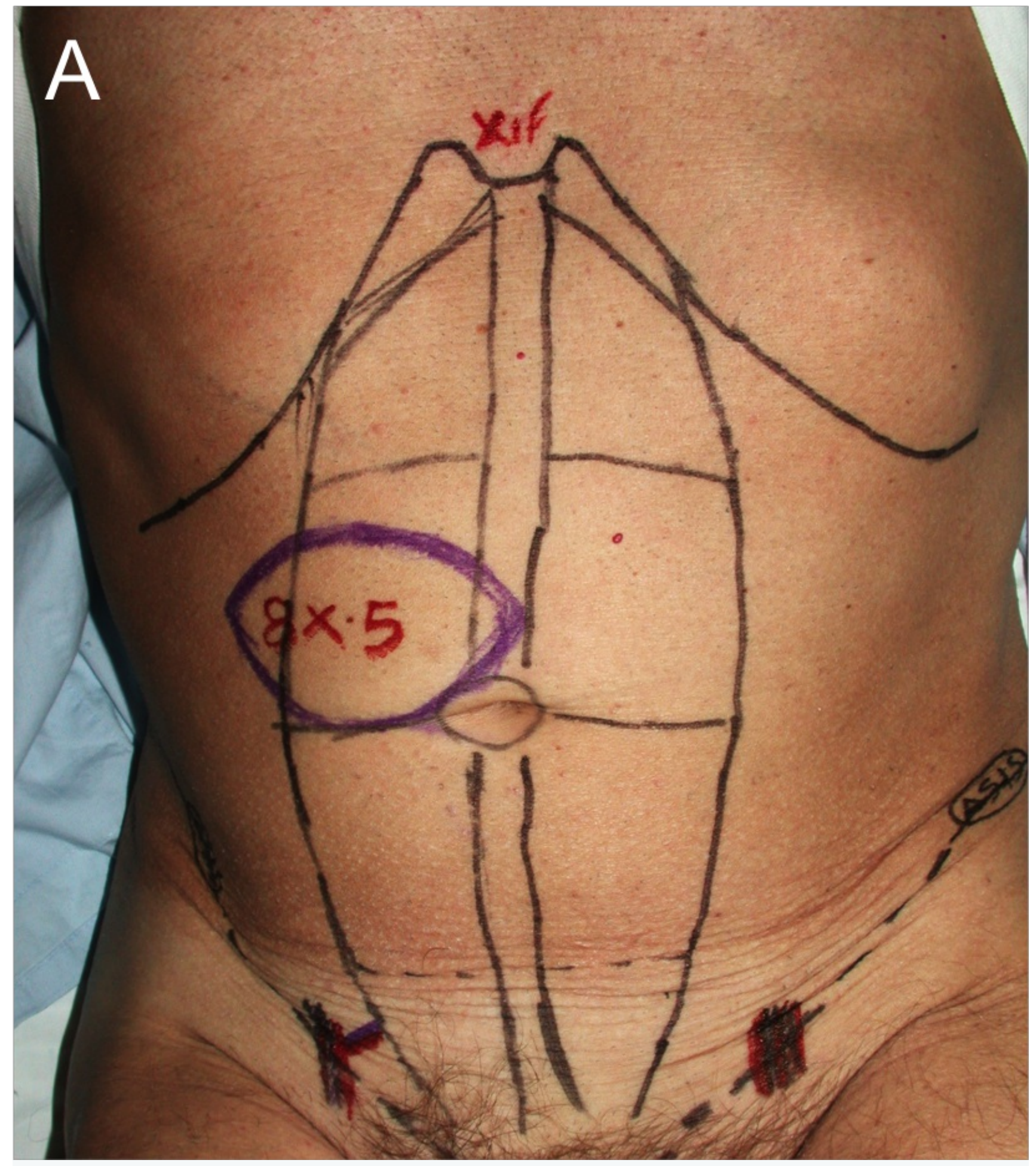

Figure 1A: Design of standard abdominal flaps for head and neck reconstruction. Horizontal TRAM flap design centered over the rectus abdominis muscle in the upper abdomen 


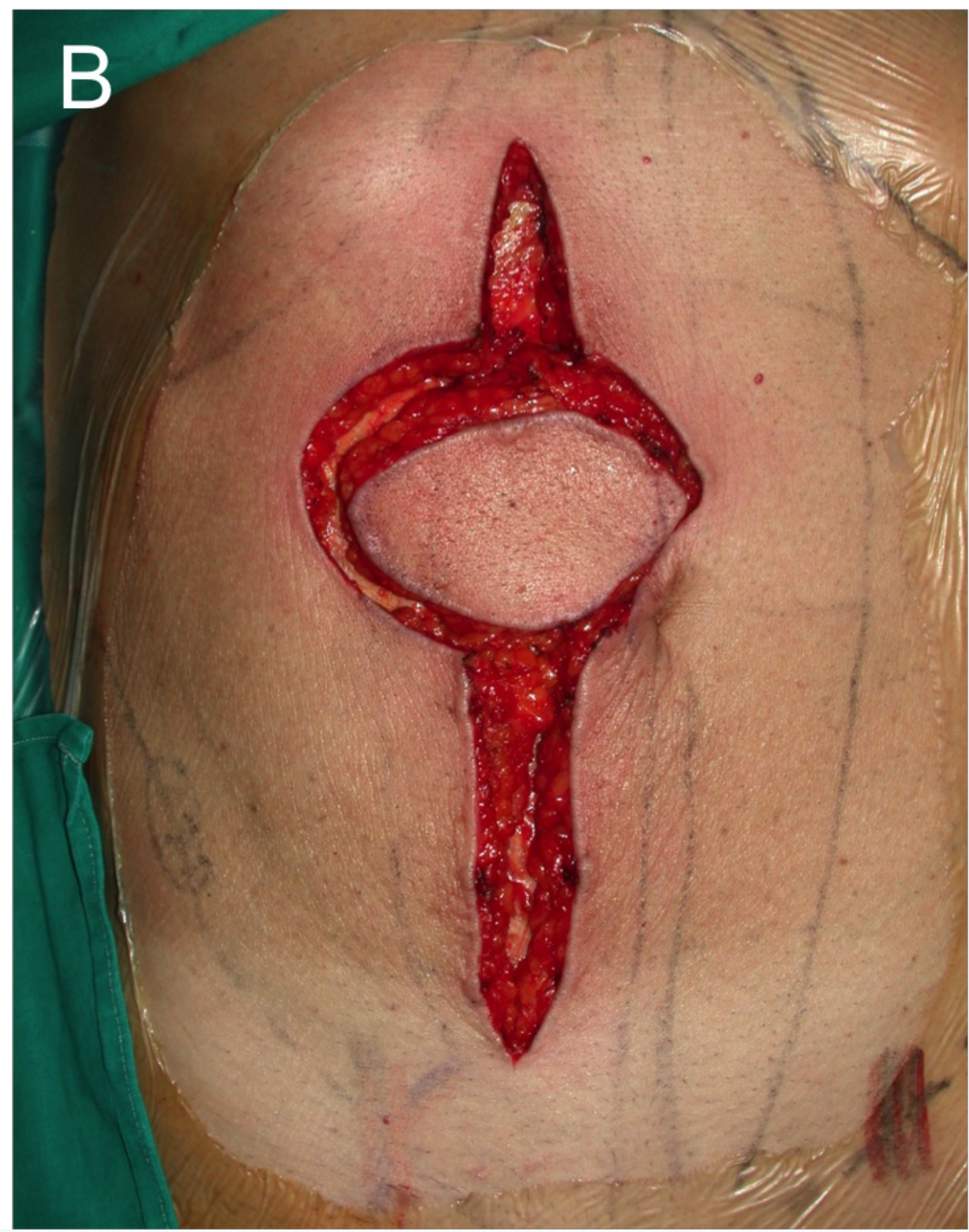

Figure 1B: Elevation of standard TRAM flap for head and neck reconstruction. The vertical skin incision encompasses both the lower and upper abdominal region to include the rectus abdominis muscle.

For bilateral harvest of flaps, the abdominal skin paddles were designed covering both sides of the inferior aspect of the anterior abdominal wall, with the patient in the supine position and the knees slightly flexed. [9]

Typically, the lower incision is transversely placed at the suprapubic crease and above the inguinal ligament up to the anterior superior iliac spines (ASIS). The upper incision is placed above the umbilicus and gently curves laterally to reach 
the ASIS. (Fig. 2B)

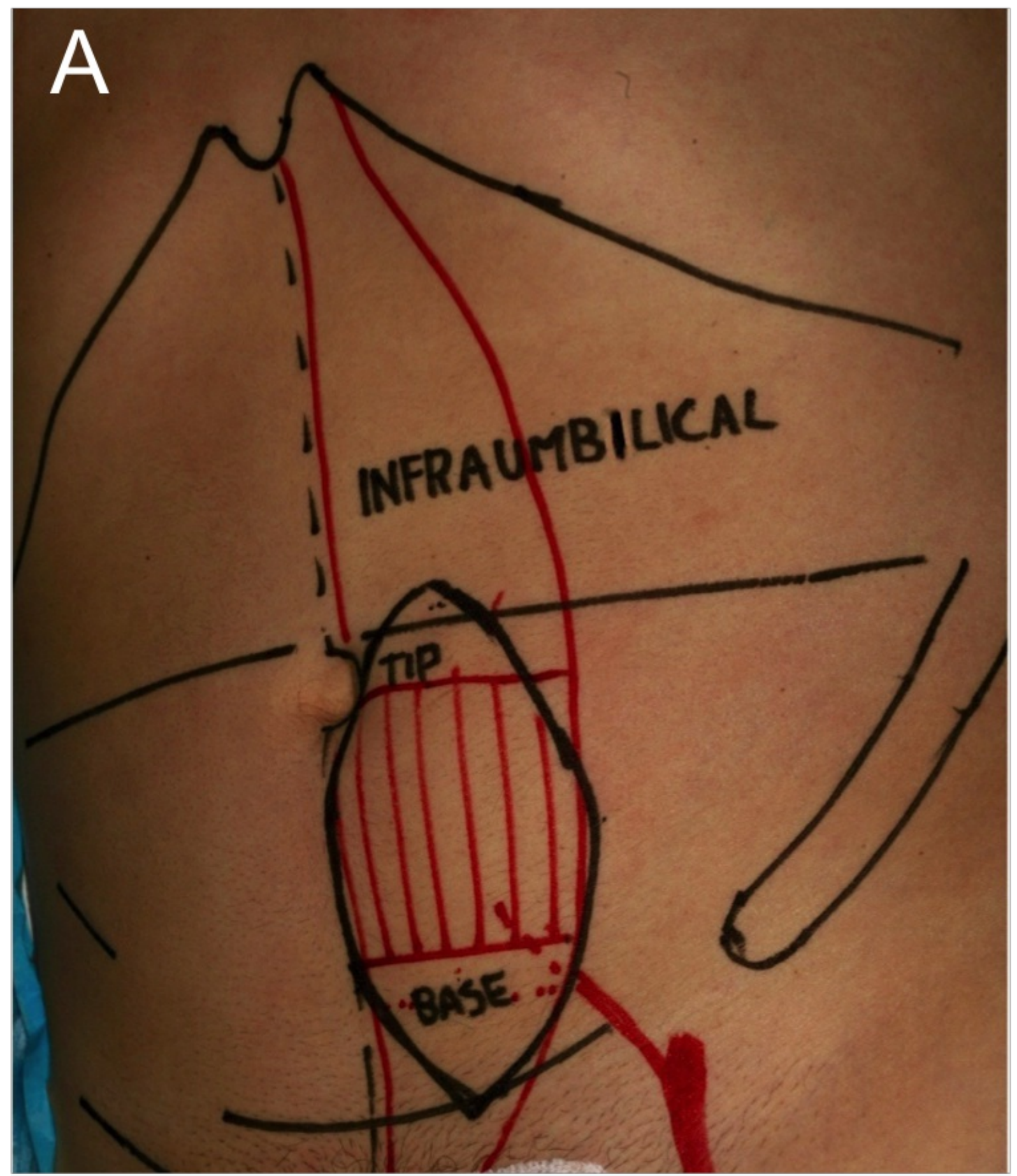

Figure 2A: Skin paddle design of infraumbilical abdominal flaps. Preoperative skin markers of left infraumbilical VRAM flap designed after standard anatomical landmarks tracking (intercostal inferior border, line crossing the umbilicus, iliac crest, linea alba and the pubic symphysis in black colored reference lines). The skin paddle was outlined along the linea alba centered on the carrier RA muscle (red grid) and extending over the lower abdominal wall between the umbilicus and the pubis. The superior aspect of the skin incision is marked shortly encompassing the umbilicus line to eventually correct an ear-dog deformity. 


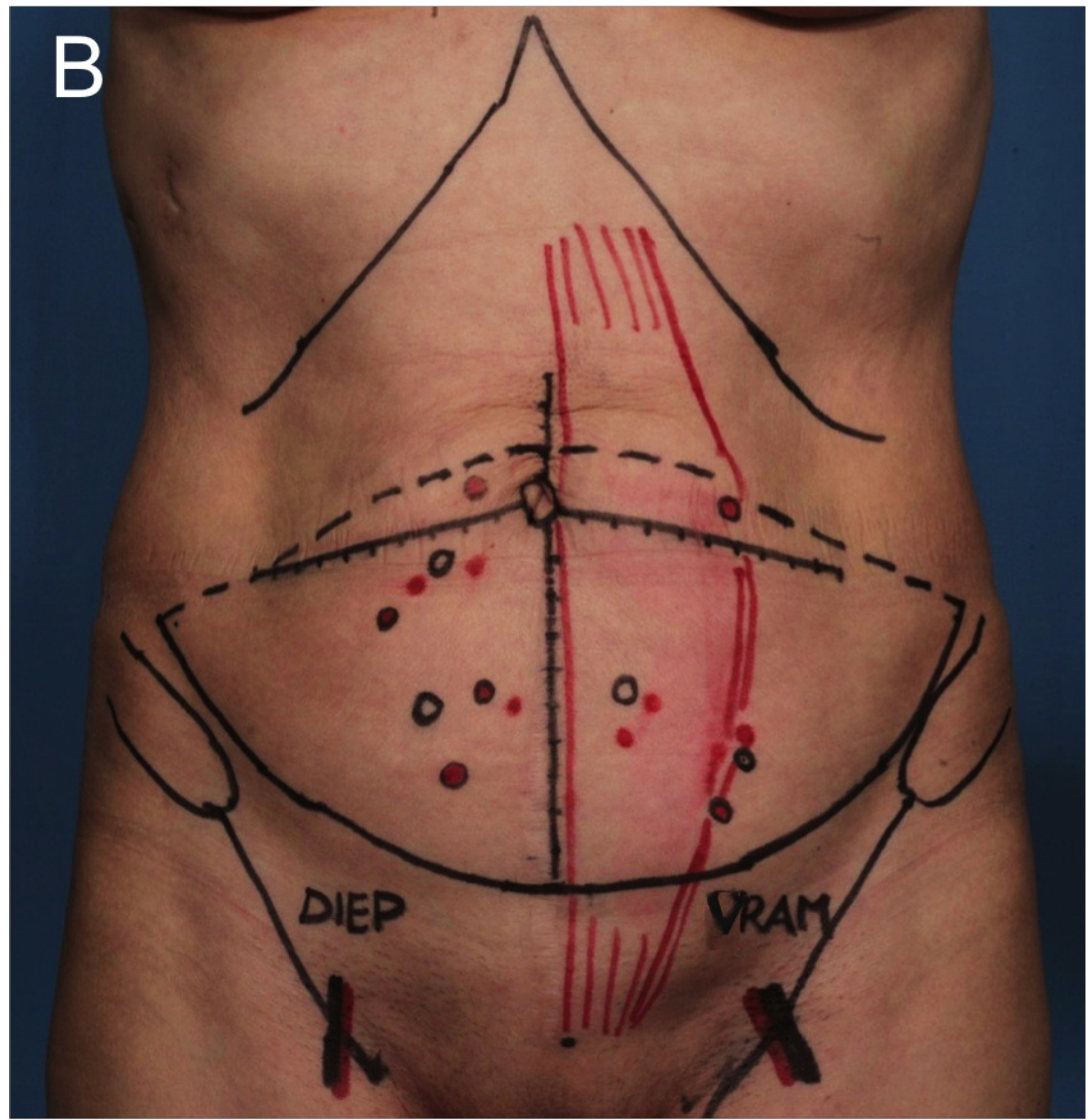

Figure 2B: Skin paddle design of infraumbilical abdominal flaps. Preoperative skin markers of bilateral infraumbilical flap (simultaneous right DIEAP and left VRAM) after standard anatomical landmarks tracking (see above). Probe detection of two-sided main pedicles and paraumbilical perforators was also outlined (main and minor vessels in black and red circles respectively).

When a VRAM flap was planned, a full width harvest of the RA muscle and anterior rectus sheet above the arcuate line was performed. Careful dissection of the deep inferior epigastric pedicle down to its origin from the iliac vessels provided a pedicle 8 to $10 \mathrm{~cm}$ long. Strengthening of the abdominal wall was achieved with the use of a synthetic non-absorbable mesh (2/0 nylon suture) to repair the muscle defect (Fig. 3A).

When a DIEAP flap was planned, the rectus sheet was opened at the selected perforator that was traced through the muscle, splitting the RA muscle fibers and preserving the intercostal nerves. The superficial inferior epigastric vein (SIEV) 
was always included as an outlet for venous congestion. A tension-free coaptation of the rectus sheet was achieved with a running large caliber non-absorbable suture (Fig. 3B).

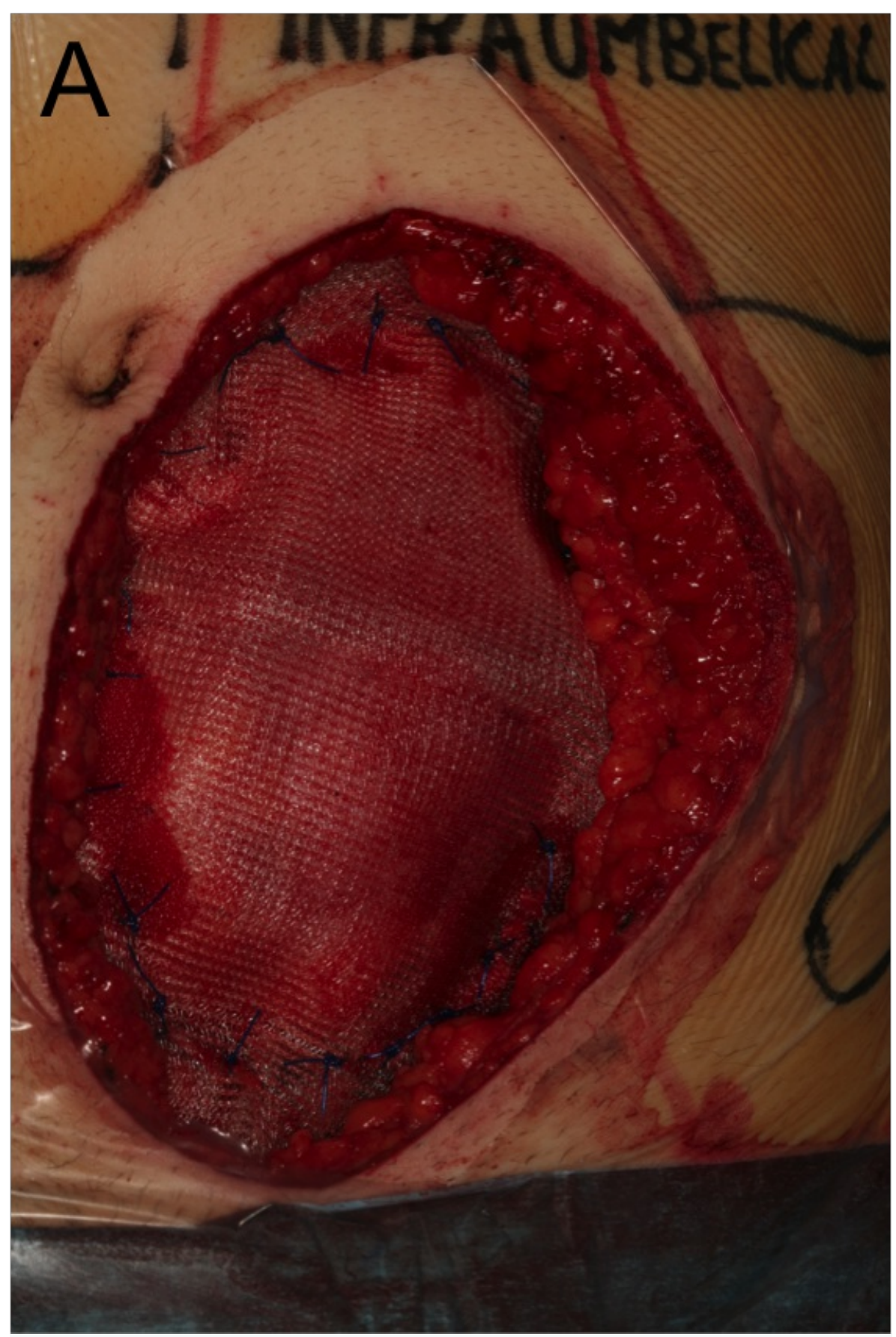

Figure 3A - Abdominal wall reconstruction.A. RA muscle repair with propylene mesh for unilateral VRAM flap harvest. 


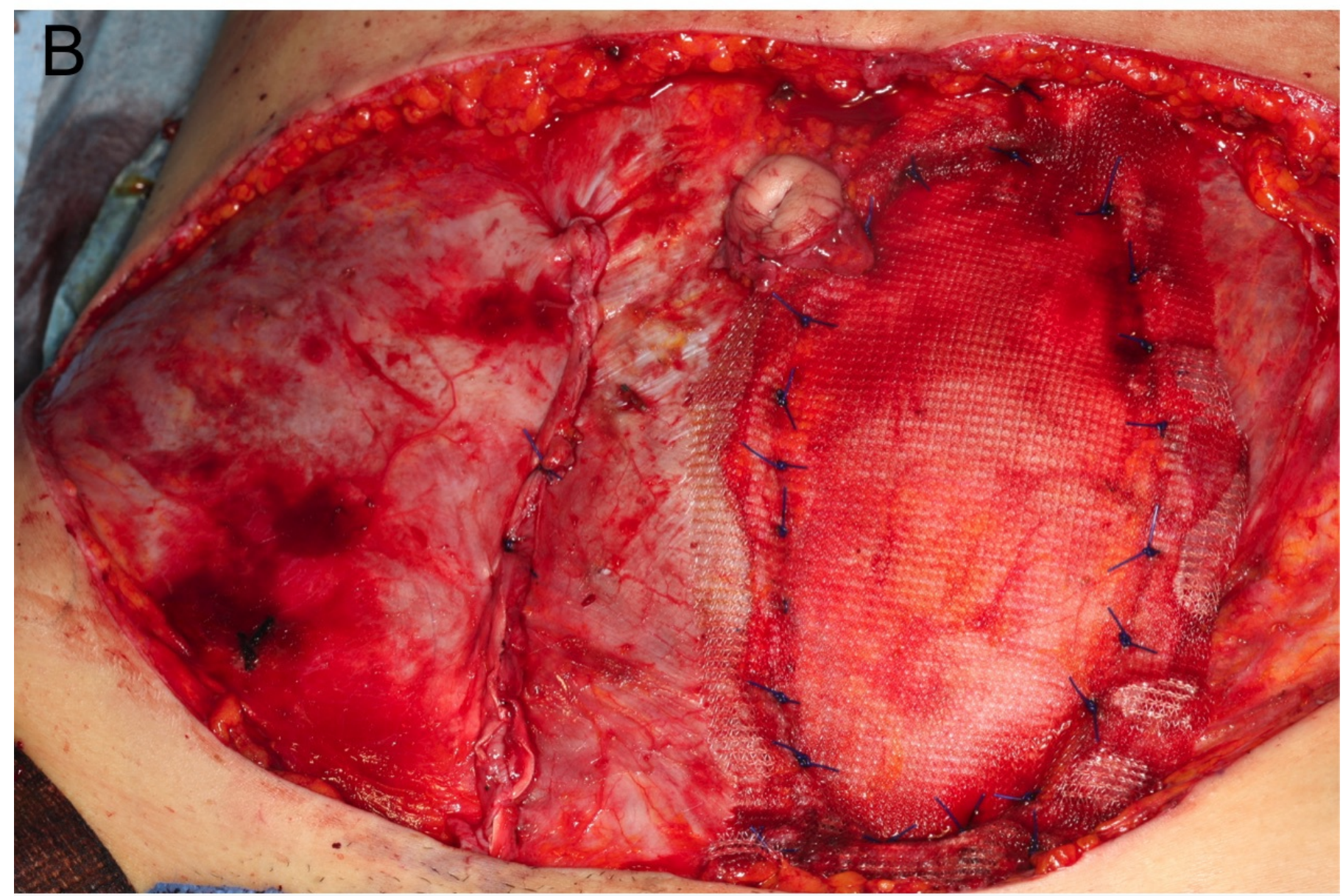

Figure 3B - Abdominal wall reconstruction. Direct closure (2/0 non-absorbable sutures) of the anterior sheet (right side) and propylene mesh repair of RA muscle defect (left side) after simultaneous elevation of a right DIEAP and a left VRAM infraumbilical flap respectively.

Direct skin closure resulting in a linear vertical scar was achieved in all cases of unilateral harvest of flaps. (Fig. 4A).

Standard abdominoplasty with umbilical repositioning was performed in all cases of bilateral harvest of flaps after surgical drain insertion. (Fig. 4B-C). 


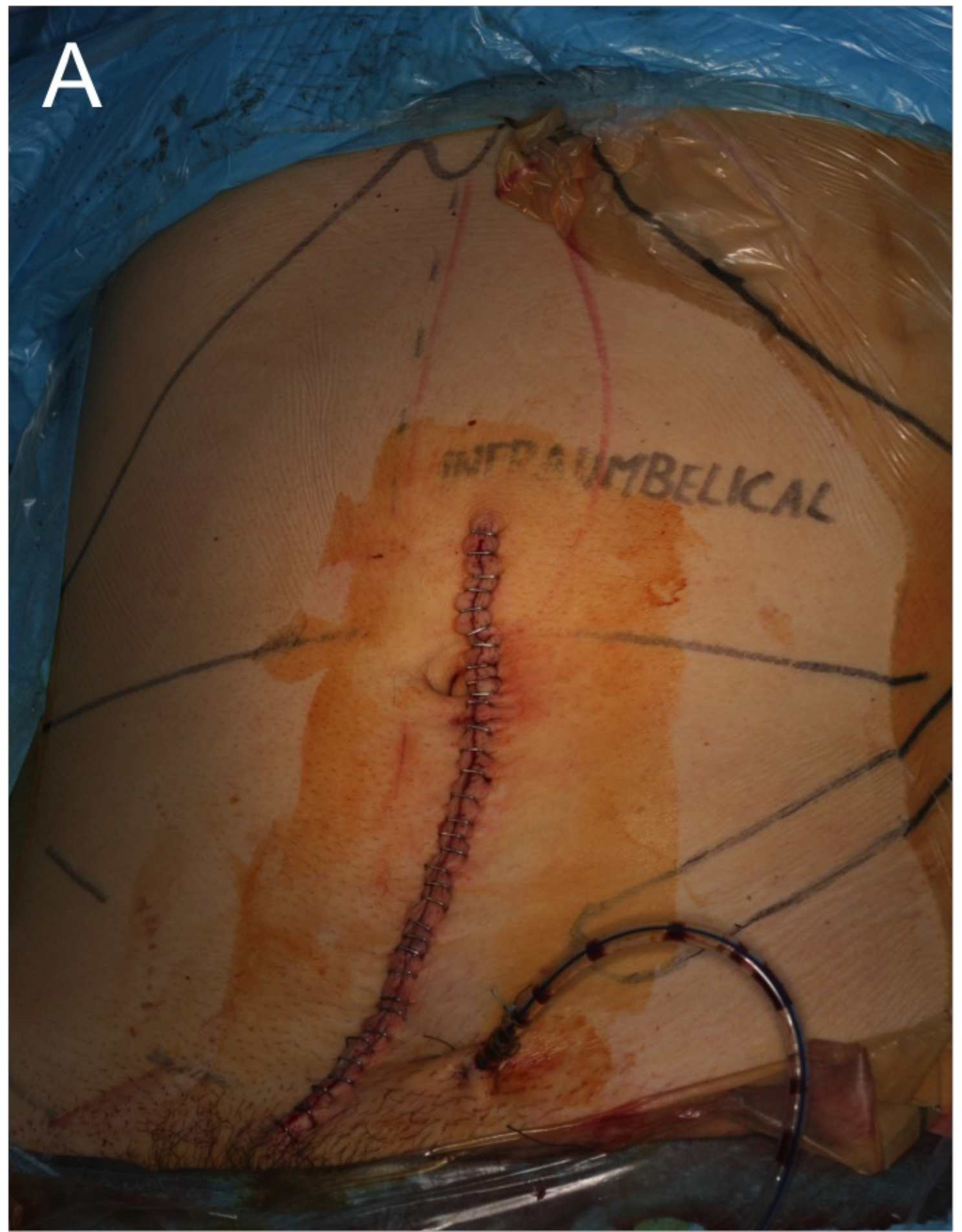

Figure 4A - Abdominal wound closure. Skin closure of the inferior abdominal region following left VRAM harvest, extending slightly above the umbilicus as correction of the residual ear dog deformity without the sacrifice of the upper abdominal skin. 


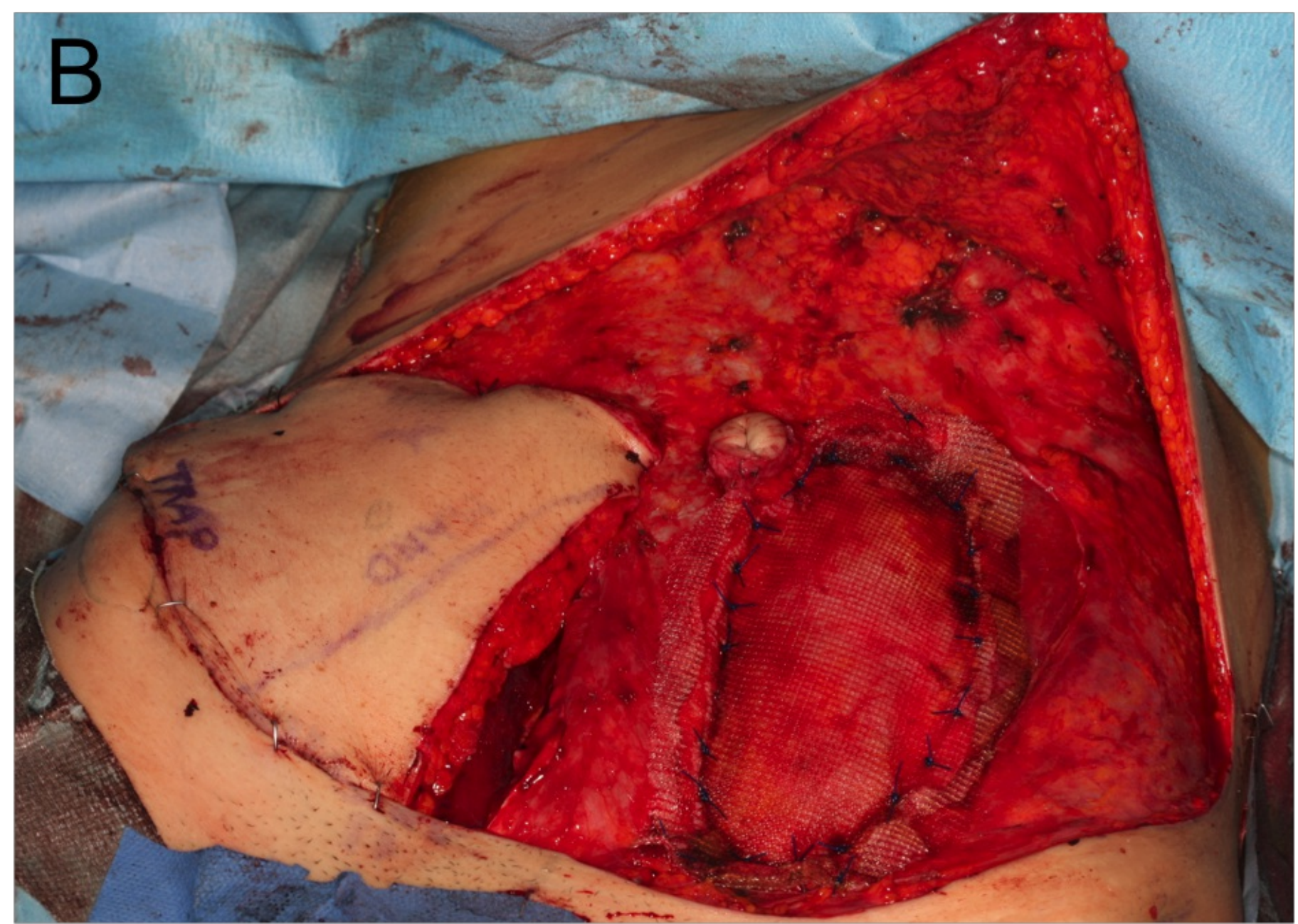

Figure 4B - Abdominal wound closure. Bilateral infraumbilical flap elevation with mesh closure of RA defect after VRAM flap inset (left side) and DIEAP flap still on site (right side). Abdominal wall superficial layers (skin, fat, and muscles) are carefully dissected for abdominoplasty preparation. 


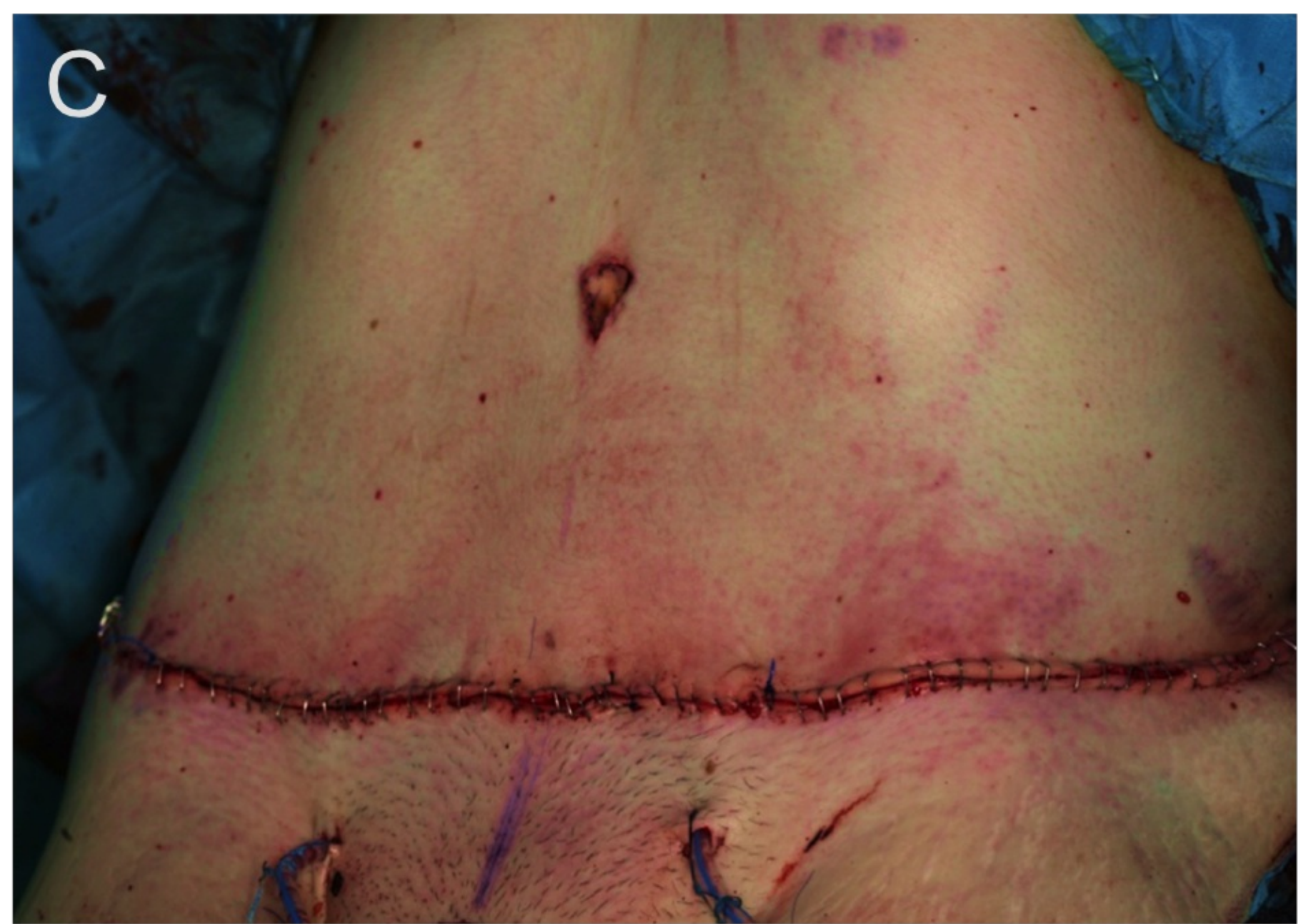

Figure 4C. Final result after standard abdominoplasty and umbilical repositioning in the same patient

\section{Postoperative follow-up}

After surgery, postoperative systemic complications, donor-site complications, need for abdominal reoperative surgery were recorded; free flap viability at the recipient site, need for additional salvage surgery in case of flap failure, and length of hospital stay were collected.

The histopathological report of the tissue specimens was reviewed and the ultimate cancer diagnosis was classified according to the current TNM staging system.

All cancer patients received a consultation at the institutional multidisciplinary head and neck oncology board to consider further indication to adjuvant treatments and oncological follow-up.

At discharge, outpatient clinical follow-up was started every 3 months over the first year, every 6 months over the second year, and annually thereafter. Data on cancer-free disease survival, abdominal wall integrity and abdominal wound healing were retrieved from the patient follow-up charts and digital pictures from the local database of the recruiting centers. Pain intensity at the donor site using a visual analogue scale (VAS) ranging from 0 (no pain) to 10 (unbearable pain) was recorded at discharge and at each follow-up visit.

At each visit, all patients were asked about any physical impairment related to abdominal wall weakness, and the donor site was inspected for any sign of anterior wall laxity and unsightly scars. 


\section{Study outcome}

The main outcome of the study was the occurrence of donor site complications in short (within 1-month), medium (6-month) and long-term follow-up (up to latest follow-up). Complications were categorized as minor or major.

Abdominal bulging and hernia formation were defined as major complications, regardless of the need for reparative surgery. Physical examination and abdominal ultrasonography were performed to confirm diagnosis.

Complicated abdominal wound healing with surgical-site infection/skin dehiscence were classified as minor when successfully managed with medications or local flap transposition. Cases requiring major reparative surgical interventions or repeated additional surgical procedures to correct abdominal skin unfavorable outcome were considered as major complications.

Abdominal wall firmness was determined by the absence of muscle hypotony with complete resumption of daily activities. The final appearance of the abdominal wall was inspected for the presence of overall symmetrical abdominal contour and stable infraumbilical scar healing without unfavorable skin remnants. A score system graded 1-3 was used to define poor, satisfactory and good results, respectively.

Different physicians (A.F., G.S., P.F., M.L.) from those who had performed surgery evaluated donor site functional and aesthetic results at each scheduled postoperative visit and recorded them on individual case report form.

Recipient site was also inspected for flap failure that was categorized as complete in presence of total flap necrosis, and partial in cases of incomplete skin loss (variable extent).

\section{Statistical analysis}

Continuous variables are reported as median $\left(50^{\text {th }}\right.$ percentile) and interquartile range (IQR, $25^{\text {th }}$ and $75^{\text {th }}$ percentiles). Discrete variables are reported as the number and proportion of subjects with the characteristic of interest. Statistical analysis was performed using Stata 16.1 (Stata Corporation, College Station, TX, USA).

\section{RESULTS}

\section{Patients}

In the period between January 2008-January 2020, 25 consecutive patients underwent reconstruction of soft tissue defects of the cervico-facial region with free flaps harvested from the lower abdomen. The baseline features of study population are given in Table 1.

The patients were 11 men and 14 women with a median (IQR) age of $56(47 ; 65)$ years.

The most frequent diagnosis was oral squamous cell carcinoma $(n=21,84 \%)$, of these 6 patients had recurrent malignancies following surgery and radiation treatment at primary cancer diagnosis. Isolated tongue defects (total glossectomy) were treated in 8 cases, whereas the remaining 17 patients needed reconstruction of composite hard and soft tissue defects in the cervico-facial region.

A total of 35 abdominal infraumbilical free flaps were performed and 10 patients underwent bilateral flap harvest.

The average soft tissue defect size was $11 \times 8 \mathrm{~cm}$ (maximum defect size $12 \times 8 \mathrm{~cm}$ ) after total glossectomy, and $18 \times 9 \mathrm{~cm}$ 
(maximum defect size $34 \times 14 \mathrm{~cm}$ ) in patients who underwent resection of cervico-facial skin respectively.

Flap necrosis occurred in 4 cases (1 VRAM, 3 DIEAP) that required salvage microsurgical reconstructive procedures. In four cases of bilateral DIEAP elevation, partial skin necrosis (10 to $40 \%$ volume in one case) required additional tissues transfer (2 free flap, 1 temporalis flap) in three patients. In the remaining patient secondary healing was achieved.

\section{Baseline features}

Sex

Women

Men

Age (years)

Diagnosis

Necrotizing ulcerative stomatitis

Osteoradionecrosis

Oral squamous cell cancer

Oral squamous cell cancer - recurrence

Osteosarcoma - recurrence
$\mathrm{N}=25$

$14(56 \%)$

$11(44 \%)$

$56(47 ; 65)$

$1(4 \%)$

$2(8 \%)$

$15(60 \%)$

$1(4 \%)$

$1(4 \%)$

$5(20 \%)$

Type of defect

Soft tissue

$13(52 \%)$

Composite defect

$12(48 \%)$

Total glossectomy

$8(32 \%)$

Cervico-facial defect

$17(68 \%)$

Type of flap

\begin{tabular}{|l|l}
\hline Monolateral & 15 \\
\hline VRAM & 12 \\
\hline DIEAP & 3 \\
\hline Bilateral & 10 \\
\hline VRAM + DIEAP & 6 \\
\hline Bilateral DIEAP & 3 \\
\hline Bilateral VRAM & 1
\end{tabular}

\section{Table 1 - Features of the patients at the baseline.}

Continuous variables are reported as median and interquartile range (IQR) and discrete variables are reported as the number and proportion of patients with the characteristic of interest.

Median hospital stay was 32 days (IQR 26; 46), and median follow-up was 12 months (IQR 6; 27). One patient died 1 month after surgery for severe systemic complication following acute respiratory distress, and seven more patients died of cancer-related disease during the follow-up. The 1 -year overall survival rate of the study population was 52\%. (Table 2) 
Systemic complications

\begin{tabular}{l|l|}
\hline None & $22(88 \%)$ \\
\hline ARDS & $1(4 \%)$ \\
\hline Lower leg ischemia & $1(4 \%)$ \\
\hline Stroke & $1(4 \%)$ \\
\hline
\end{tabular}

Follow-up (months) $\quad 12(6 ; 27)$

pTNM (22 cancer patients)

\begin{tabular}{|l|l|}
\hline Early stage $\left(\mathrm{T}_{1,2} \mathrm{~N}_{0}\right)$ & 3 \\
\hline Advanced stage $\left(\mathrm{T}_{2,3,4}\right.$ every $\left.\mathrm{N}\right)$ & 19 \\
\hline
\end{tabular}

$\mathrm{N}=35$

Free flap survival

\begin{tabular}{l|l}
\hline Yes & $27(77 \%)$ \\
\hline Total necrosis & $4(11 \%)$ \\
\hline Partial necrosis (10\%) & $2(6 \%)$ \\
\hline Partial necrosis (20\%) & $1(3 \%)$ \\
\hline Partial necrosis $(40 \%)$ & $1(3 \%)$ \\
\hline Salvage surgery & \\
\hline None & $28(80 \%)$ \\
\hline Free flap & $5(14 \%)$ \\
\hline Pedunculated flap & $2(6 \%)$
\end{tabular}

Donor site

VAS $\quad 0(0 ; 1)$

Complications

\begin{tabular}{l|l}
\hline None & $31(88 \%)$ \\
\hline Marginal necrosis (partial) & $2(6 \%)$ \\
\hline Periumbilical necrosis (partial) & $1(3 \%)$ \\
\hline Bulge (VRAM) & $1(3 \%)$
\end{tabular}

Reoperative surgery

\begin{tabular}{l|l} 
None & $34(97 \%)$ \\
\hline Local flap (marginal necrosis) & $1(3 \%)$ \\
\hline Length of stay (days) & $32(26 ; 46)$
\end{tabular}

Table 2 - Postoperative features of the patients.

Continuous variables are reported as median and interquartile range (IQR) and discrete variables are reported as the number and proportion of patients with the characteristic of interest.

ARDS: Acute respiratory distress syndrome

Perioperative donor site complications 
Partial necrosis of the repositioned umbilicus occurred in one patient, and superficial abdominal seroma formation in another one that healed spontaneously in both cases. Two patients experienced marginal abdominal skin necrosis. (Fig.

5) In one case surgical correction of the necrosis required a local rotation flap, while the other case healed with mechanical debridement of non-viable tissue and topical therapies. The median (IQR) post-operative pain score recorded by VAS at the donor site was $0(0 ; 1)$.

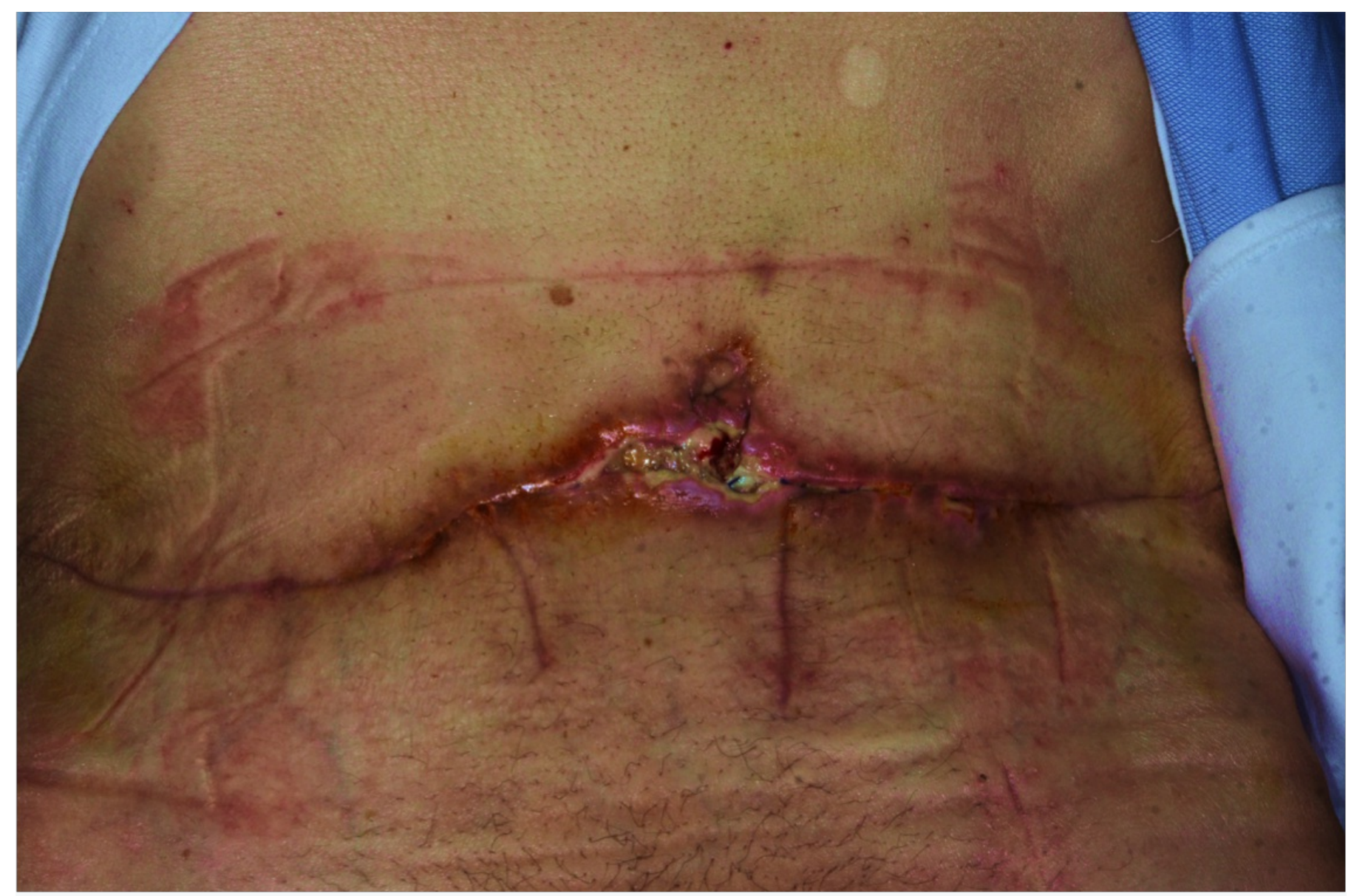

Figure 5 - Donor site short-term minor complication. Marginal periumbilical skin necrosis that occurred in a patient with severe peripheral arterial occlusive disease.

\section{Postoperative donor site complications}

Abdominal bulging occurred 1 year after surgery in one case following a two-sided flap harvest (combined VRAM/DIEAP flaps). The latter complication did not require surgical repair. (Fig. 6A-B). 




Figure 6A-Abdominal bulge. 1-y postoperative frontal view of a patient who developed abdominal bulging after the simultaneous harvest of a DIEAP flap (right side) and a VRAM flap (left side). 


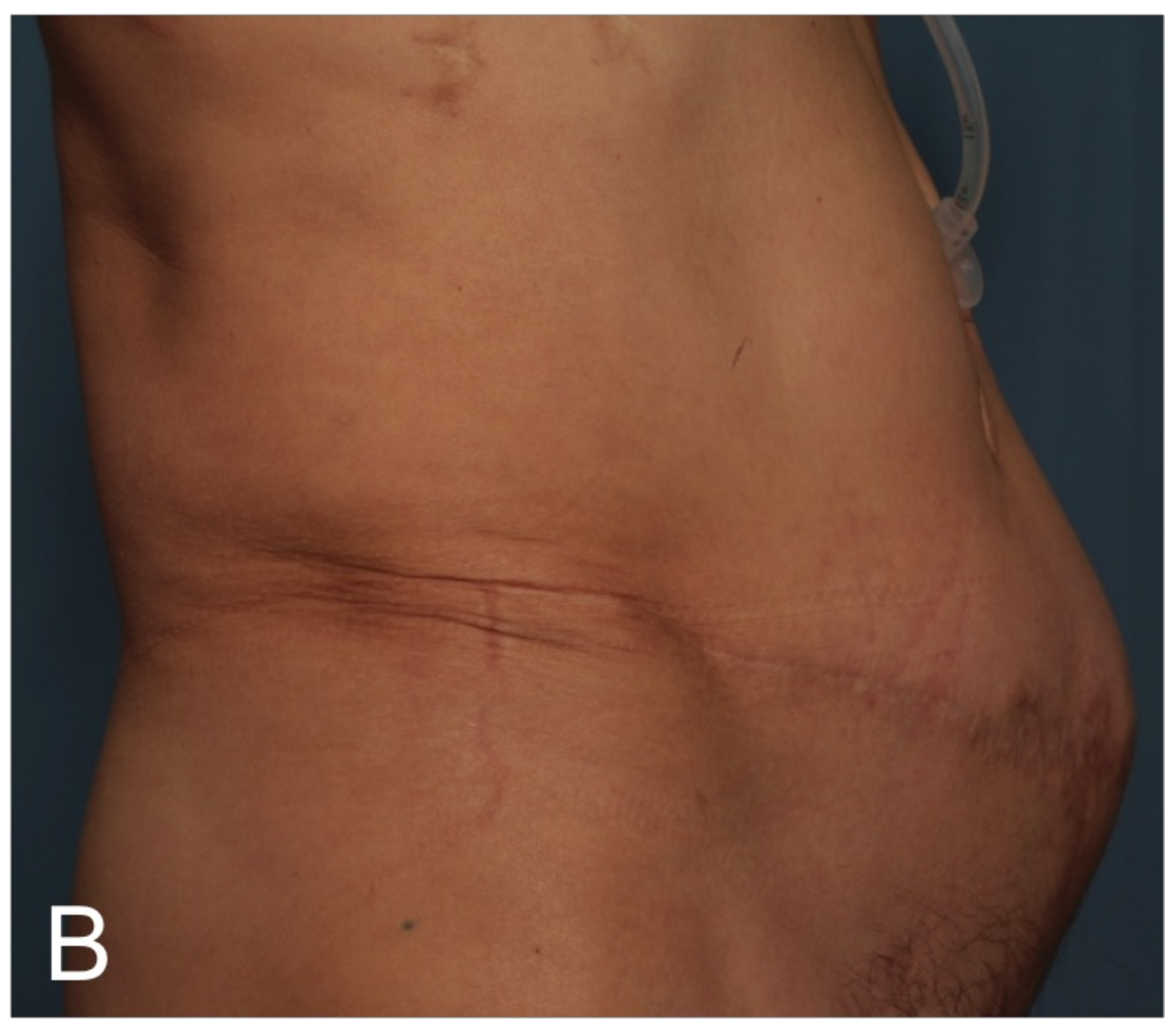

Figure 6A- Abdominal bulge. 1-y postoperative lateral view of the same patient.

Neither hernia nor abdominal wall weakness was observed for all the duration of the follow-up with complete resumption of daily activities in all patients in the short-term (within 1 month). The overall rate of postoperative abdominal complications (major/minor) was $11 \%$.

The final appearance of the abdominal wall was judged good (grade 3) in all but two patients (Fig. 7A-B). 


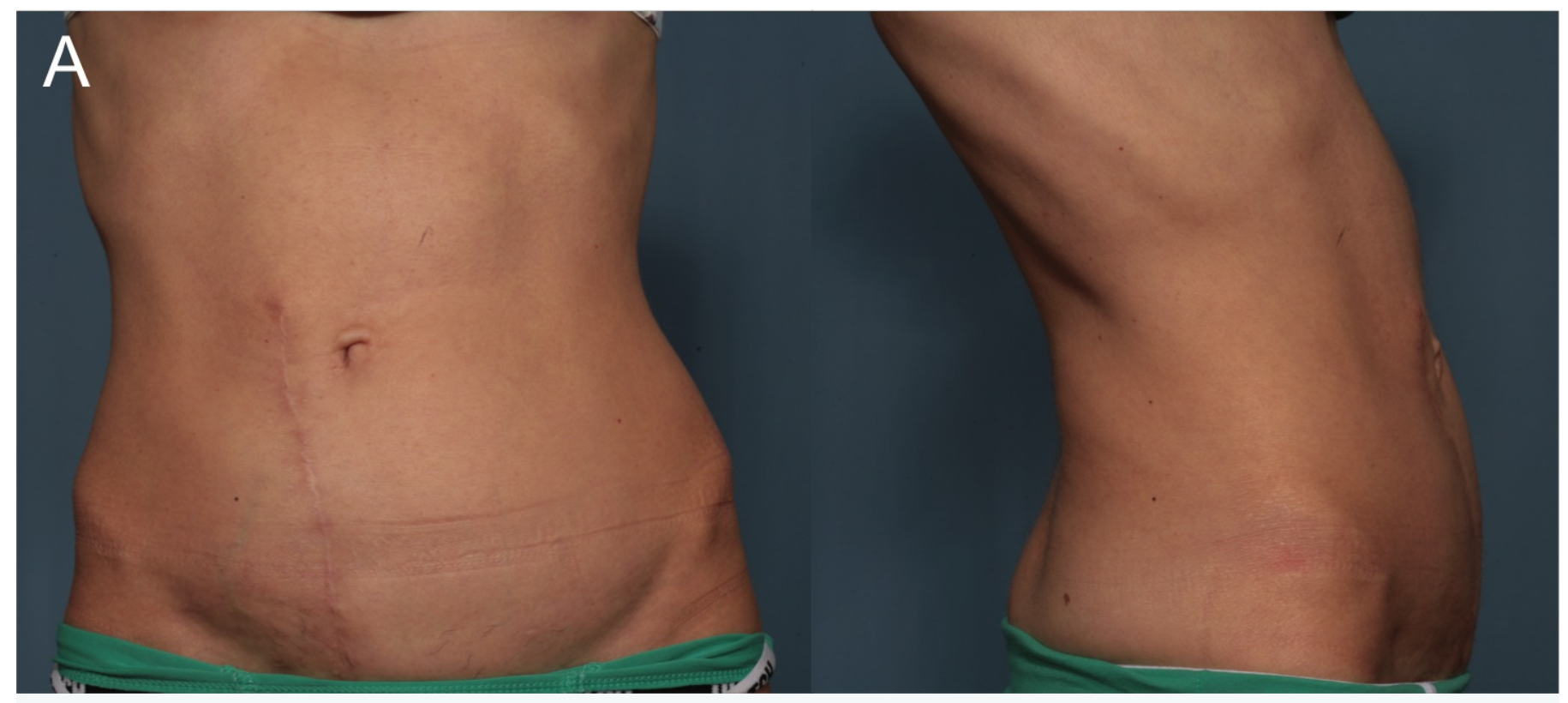

Figure 7A - Donor site long-term follow-up. Frontal and lateral view of a woman who underwent a right infraumbilical VRAM harvest. The abdominal wall contour is acceptable as compared with the contralateral untreated side with the skin scar entirely located in the lower abdomen.

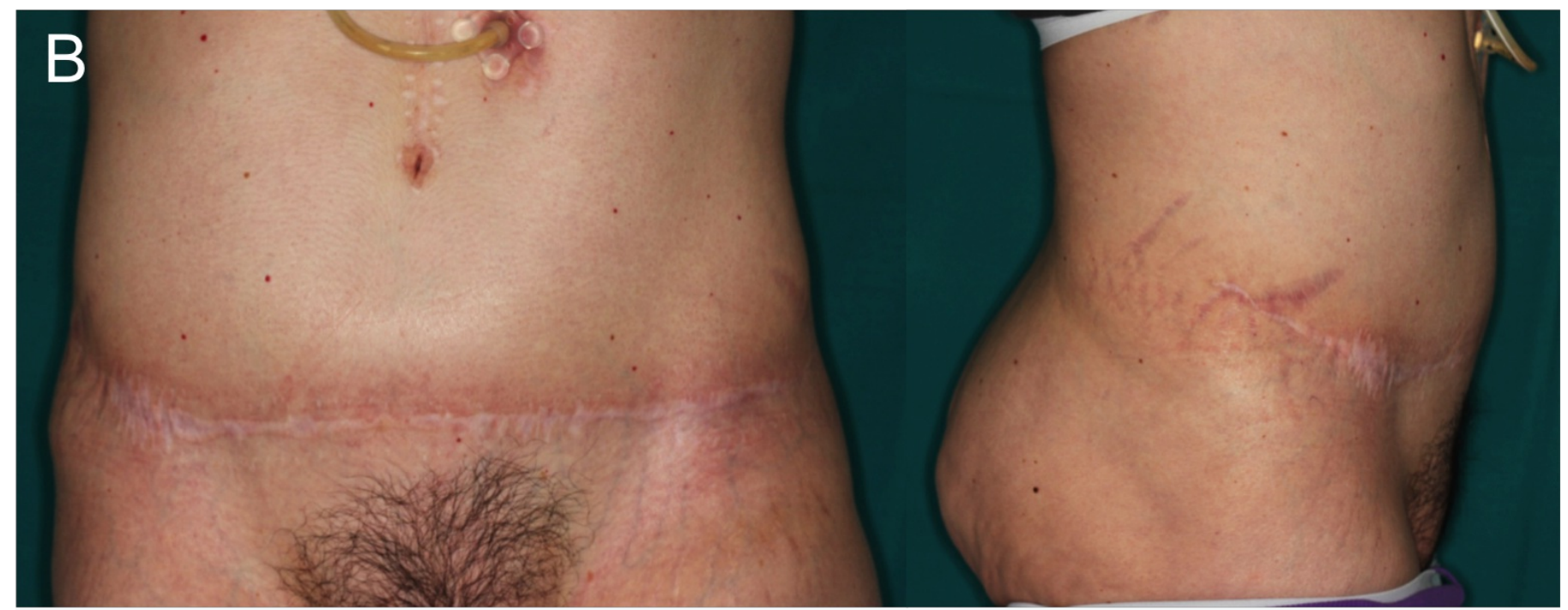

Figure 7B - Donor site long-term follow-up. Frontal and lateral view of a male patient who underwent abdominoplasty flap closure and relocation of the umbilicus following bilateral infraumbilical DIEAP flap harvest

\section{DISCUSSION}

The present cohort study shows the feasibility and safety of the infraumbilical harvest of soft-tissue flaps in terms of functional and esthetic results at the donor site. The harvest of bilateral flaps with the inclusion of a segment of RA muscle above the arcuate line does not endanger the strength of the abdominal wall, provided that accurate reconstruction of the missing layers is accomplished.

The main study limitation is the retrospective nature of the data collected. However, consecutive cases were enrolled with different defect and flap size accordingly.

A second limitation is the unavailability of postoperative instrumental evaluation of abdominal muscle dynamics (i.e. 
electromyography) as completion of the donor-site morbidity assessment.

This is, however, largely due to the fact that we enrolled mostly advanced-stage cancer-patients (primary or recurrent malignancy) who were recommended for adjuvant therapies and/or long-term supportive care (enteral feeding) after surgery.

The reconstruction of head and neck defects is often challenging for the functional implications of the three-dimensional composite framework of the cervico-facial area and usually required great amount of donor tissues.

Abdominal perforator flaps based on DIEA vascular territory have become an important donor source of large volume of vascularized tissues that can be tailored to the defective recipient site in the cervico-facial area. ${ }^{[1][3][10][11]}$

TRAM/VRAM flap is commonly used to reconstruct subtotal/total glossectomy defects providing highly versatile tissues supporting the restoration of bulky defects. ${ }^{[12][13][14]}$ Instead, DIEAP adipocutaneous flaps can be used to cover large cutaneous defects of the cheek and the neck. [2][7][15][16]

The standard design of the abdominal perforator flaps (TRAM/VRAM and DIEAP) encompasses the upper and lower aspect of the abdomen in the periumbilical area, thus including the dominant perforators to increase flap viability. [6][17] The skin component of the VRAM/TRAM flap can be oriented vertically, obliquely or transversely to include large tissue volumes, comprising the RA muscle, for reconstructive purposes.

Nevertheless, the traditional paraumbilical flap is usually associated with poor aesthetic outcomes, resulting from scar that exceeds the umbilicus in the upper abdomen. ${ }^{7}$

In our study, the infraumbilical harvest of abdominal perforators flaps showed a number of advantages over the standard technique. First, in the case of large volume defects, simultaneous harvest of bilateral adipocutaneous and musclecontaining flaps was feasible, without impacting the abdominal wall firmness. Closure by standard abdominoplasty was achieved in all patients undergoing bilateral flap harvest.

Interestingly, the harvest of a RA muscle flap component (DIEAP chimeric flap or VRAM flap) from the infraumbilical region without exceeding the arcuate line provided successfully restoration of total glossectomy defects.

Secondly, in case of flap failure following unilateral harvest of an infraumbilical flap, the contralateral side of the abdomen is readily available for the harvest of a new flap with the same features. The abdominal skin scar of the failed flap can be resected and donor site closure achieved with standard abdominoplasty technique.

On the contrary, violation of both the upper and lower abdominal skin when using standard abdominal flap design precludes the use of the contralateral side in case of flap failure.

Third, the infraumbilical approach, in both transverse and vertical design (unilateral or bilateral), allows for a more concealable scar located in the inferior aspect of the abdomen.

Of importance, the pedicle length of the infraumbilical flaps was appropriate in all cases for the reconstructive purpose, including secondary reconstruction of large soft-tissue defects in vessel-depleted neck due to tumor extirpation and radiotherapy.

In our study, the rate of post-operative abdominal complications was in line with others studies reporting the donor site 
morbidity of standard periumbilical flap harvest (range 3.5-20\%). [18][19][20]

In conclusion, our study supports the hypothesis that the infraumbilical area is a reliable donor region of free flaps for the reconstruction of composite defects of the head and neck, Harvest of multiple flaps (sequentially or simultaneously) from a single donor area does not preclude abdominal wall integrity.

Placing the abdominal flaps entirely in the lower abdomen does not impact the abdominal wall firmness and provides satisfactory functional and morphological results at the donor site.

\section{- $\quad$ Funding}

This research did not receive any specific grant.

\section{References}

1. ${ }^{a}, \mathrm{~b}$ Peter C. Neligan. (2013). Head and Neck Reconstruction. Plastic and Reconstructive Surgery, vol. 131 (2), 260e269e. doi:10.1097/prs.0b013e3182778938.

2. ${ }^{a, b}$ Eamon S. Beausang, Doug McKay, Dale H. Brown, Jonathan C. Irish, Ralph Gilbert, Patrick J. Gullane. (2003). Deep Inferior Epigastric Artery Perforator Flaps in Head and Neck Reconstruction. Annals of Plastic Surgery, vol. 51 (6), 561-563. doi:10.1097/01.sap.0000096447.93223.bf.

3. a, bJohn W. Frederick, Larissa Sweeny, William R. Carroll, Glenn E. Peters, Eben L. Rosenthal. (2013). Outcomes in head and neck reconstruction by surgical site and donor site. The Laryngoscope, vol. 123 (7), 1612-1617. doi:10.1002/lary.23775.

4. a, bJ. Brian Boyd, G. Ian Taylor, Russell Corlett. (1984). The Vascular Territories of the Superior Epigastric and the Deep Inferior Epigastric Systems. Plastic and Reconstructive Surgery, vol. 73 (1), 1-14. doi:10.1097/00006534$198401000-00001$.

5. ^ P.N. Blondeel. (1999). One hundred free DIEP flap breast reconstructions: a personal experience. British Journal of Plastic Surgery, vol. 52 (2), 104-111. doi:10.1054/bjps.1998.3033.

6. ${ }^{a}$, bW.M. Rozen, M.W. Ashton, G.I. Taylor. (2008). Reviewing the vascular supply of the anterior abdominal wall: Redefining anatomy for increasingly refined surgery. Clin. Anat., vol. 21 (2), 89-98. doi:10.1002/ca.20585.

7. a, b, c Jaume Masià, Maria Sommario, Daniele Cervelli, Carmen Vega, Xavier León, Gemma Pons. (2010). Extended deep inferior epigastric artery perforator flap for head and neck reconstruction: A clinical experience with 100 patients. Head Neck, vol. 33 (9), 1328-1334. doi:10.1002/hed.21628.

8. 'Bouraoui Kotti. (2014). Optimizing the Pedicled Rectus Abdominis Flap: Revised Designs and Vascular Classification for Safer Procedures. Aesth Plast Surg, vol. 38 (2), 387-394. doi:10.1007/s00266-014-0273-y.

9. ^Phillip N. Blondeel, K.H. Van Landuyt, S.J. Monstrey. (1999). Surgical-technical aspects of the free diep flap for breast reconstruction. Operative Techniques in Plastic and Reconstructive Surgery, vol. 6 (1), 27-37. doi:10.1016/s10710949(99)80017-1.

10. ^Neal D Futran, Eduardo Mendez. (2006). Developments in reconstruction of midface and maxilla. The Lancet 
Oncology, vol. 7 (3), 249-258. doi:10.1016/s1470-2045(06)70616-7.

11. 'Stephen Y. Kang, Matthew E. Spector, Douglas B. Chepeha. (2017). Perforator based rectus free tissue transfer for head and neck reconstruction: New reconstructive advantages from an old friend. Oral Oncology, vol. 74, 163-170. doi:10.1016/j.oraloncology.2017.06.029.

12. 'Nicholas Haddock, Mark DeLacure, Pierre Saadeh. (2008). Functional Reconstruction of Glossectomy Defects: The Vertical Rectus Abdominus Myocutaneous Neotongue. J reconstr Microsurg, vol. 24 (05), 343-350. doi:10.1055/s-20081080537.

13. `Jose María López-Arcas, Javier Arias, María José Morán, Ignacio Navarro, Lorena Pingarrón, Manuel Chamorro. (2012). The Deep Inferior Epigastric Artery Perforator (DIEAP) Flap for Total Glossectomy Reconstruction. Journal of Oral and Maxillofacial Surgery, vol. 70 (3), 740-747. doi:10.1016/j.joms.2011.02.098.

14. `Johnny Cappiello, Cesare Piazza, Valentina Taglietti, Piero Nicolai. (2011). Deep inferior epigastric artery perforated rectus abdominis free flap for head and neck reconstruction. Eur Arch Otorhinolaryngol, vol. 269 (4), 1219-1224. doi:10.1007/s00405-011-1749-x.

15. ^Bradford A. Woodworth, M. Boyd Gillespie, Terry Day, Richard M. Kline. (2006). Muscle-sparing abdominal free flaps in head and neck reconstruction. Head Neck, vol. 28 (9), 802-807. doi:10.1002/hed.20393.

16. ^Bin Zhang, De-zhi Li, Zhen-gang Xu, Ping-zhang Tang. (2009). Deep inferior epigastric artery perforator free flaps in head and neck reconstruction. Oral Oncology, vol. 45 (2), 116-120. doi:10.1016/j.oraloncology.2008.04.005.

17. ^Nima P. Patel, Evan Matros, Peter G. Cordeiro. (2012). The Use of the Multi-Island Vertical Rectus Abdominis Myocutaneous Flap in Head and Neck Reconstruction. Annals of Plastic Surgery, vol. 69 (4), 403-407. doi:10.1097/sap.0b013e3182400ed8.

18. `Derrick C. Wan, Charles Y. Tseng, John Anderson-Dam, Andrew L. Dalio, Christopher A. Crisera, Jaco H. Festekjian. (2010). Inclusion of Mesh in Donor-Site Repair of Free TRAM and Muscle-Sparing Free TRAM Flaps Yields Rates of Abdominal Complications Comparable to Those of DIEP Flap Reconstruction. Plastic and Reconstructive Surgery, vol. 126 (2), 367-374. doi:10.1097/prs.0b013e3181de1b7e.

19. ' Li-Xing Man, Jesse C. Selber, Joseph M. Serletti. (2009). Abdominal Wall following Free TRAM or DIEP Flap Reconstruction: A Meta-Analysis and Critical Review. Plastic and Reconstructive Surgery, vol. 124 (3), $752-764$. doi:10.1097/prs.0b013e31818b7533.

20. 'Mario Rietjens, Francesca De Lorenzi, Manconi Andrea, Jean-Yves Petit, Prakasit Chirappapha, Alaa Hamza. (2015). Technique for Minimizing Donor-site Morbidity after Pedicled TRAM-Flap Breast Reconstruction. Plastic and Reconstructive Surgery - Global Open, vol. 3 (8), e476. doi:10.1097/gox.0000000000000451. 